\title{
Prognostic value of the optic nerve sheath thickness as an indicator of intracranial pressure among acute stroke patients
}

\author{
Kaan Celik · Tamer Colak $\cdot \quad$ Beliz Oztok Tekten
}

Department of Emergency, Bolu Abant Izzet Baysal University, Medical Faculty, Bolu, Turkey

\section{ABSTRACT}

Aim: To evaluate the clinical value of optic nerve sheath thickness (ONST) in stroke patients.

Method: The present study was prospectively performed on 386 stroke patients who were admitted to Emergency Department, and 75 healthy volunteers of similar age and gender groups. The following criteria were evaluated for each patient: age, gender, comorbidities, neurological deficit levels [Glasgow Coma Scale (GCS), the National Institute of Health Sciences Scale (NIHSS)], tomography findings (hemorrhagic / ischemic), ONST diameter in ultrasound scan, hospitalization and mortality rates.

Results: The median ONST value was $5.5 \mathrm{~mm}$ (IQR: 0.30) for the hemorrhagic stroke patients, and $5.25 \mathrm{~mm}$ (IQR: 0.20 ) for the ischemic stroke patients. The median ONST value of the stroke patients was significantly higher. Also, the ONST values of the hemorrhagic stroke patients were found to be significantly higher. In our study, the area below the curve was 0.865 . For the $4.5 \mathrm{~mm}$ cut-off value, the sensitivity was $96.1 \%$ and specificity was $82.7 \%$.

Conclusions: The results of our study showed that the ONST increased in the stroke patients and this increase was higher in the hemorrhagic stroke patients. We suggest that the treatment can be considered to decrease intracranial pressure if there is an increase in ONST in stroke patients.

Keywords: Stroke, optic nerve sheath thickness, ultrasound, emergency medicine.

(C) 2020 experimentalbiomedicalresearch.com

$\triangle$ Dr. Tamer Colak

Department of Emergency, Bolu Abant Izzet Baysal University, Medical Faculty, Bolu, Turkey

E mail: drtamercolak@gmail.com

Received: 2020-04-08 / Accepted: 2020-05-06

Publication Date: 2020-06-13

\section{Introduction}

Stroke is the third most common cause of mortality, and the most common cause of morbidity worldwide [1]. Among all stroke patients, $80-87 \%$ are ischemic, whereas remaining patients are hemorrhagic $[2,3]$.
Intracranial pressure (ICP) arises from pressure of the cerebrospinal fluid, blood and brain tissue. ICP can increase due to cranial trauma, stroke, the presence of a mass and infection; due to both primary reasons and the released mediators. This increase in ICP increases morbidity and mortality [4].

Emergency services commonly use imaging methods such as computed tomography (CT) and magnetic resonance imaging (MRI) for the diagnosis of stroke and the determination of ICP; however, the use of these methods is very 
limited due to the potentially unstable condition of the patients, the low sensitivity and specificity of these methods regarding ICP in many patients, and the low reproducibility [5]. ICP needs to be quickly determined and lowered as it can increase mortality. The most reliable measurement of ICP is through invasive procedures; however, the associated complications (including coagulopathy and local infections) and the requirement of special devices make it inefficient to use this method in the ER setting [6]. Therefore, researchers are constantly trying to develop new methods for the determination of ICP.

The optic nerve is part of the central nervous system; it is covered with dura mater and encompassed with the subarachnoid CSF [6]. It has been reported that increasing ICP increases the thickness of the optic nerve sheath (ONST), and that this correlation is significant [7-9]. This expansion can be measured specifically clearly at the retrobulbar level [10]. Therefore, in this prospective study, we aimed to investigate the potential value of ONST in the approach to diagnosis and treatment as an indicator of increased ICP in stroke patients admitted to our emergency clinic.

\section{Materials and methods}

The present study was prospectively performed on 386 stroke patients who were admitted to Emergency Department of a public hospital in Turkey between 01.02.2018 and 30.05.2018, and 75 healthy volunteers of similar age and gender groups. This study was approved by the Ethical Committee of Bolu Izzet Baysal University Faculty of Medicine (Date: 08/02/2018; Decision number: 2018/12). The rights of all participants were protected and written informed consents were obtained before the study according to the Helsinki Declaration. The following criteria were evaluated for each patient: age, gender, comorbidities, neurological deficit levels [Glasgow Coma Scale (GCS), the National Institute of Health Sciences Scale (NIHSS)], tomography findings (hemorrhagic/ischemic), duration to ultrasound scan [the period between referral and ultrasound (US) scan], ONST diameter in US, hospitalization and mortality rates. NIHSS and GCS score were used to determine neurological deficit levels. The main reason why US screening is preferred in this study is the ease of application at the bedside in the emergency room. In addition, absence of radiation and repeatability may provide use of patient followup. Moreover, value of US scan was tried to be supported by comparing the OSNDs measured by US scan and brain tomography.

The exclusion criteria were as follows: patients with transient ischemic attack (TIA), patients aged under 18 , vascular dementia, patients with liver failure or chronic renal insufficiency, hypoglycemia, pregnant and nursing women, patients with trauma, traumatic optic neuropathy, optic neuritis, optic nerve arachnoid cyst, and patients with orbital/cavernous sinus cysts.

The patients with conditions that may cause intracranial pressure increase including hydrocephalus, arachnoid cyst, hypertensive encephalopathy, pseudotumor cerebri, intracranial tumor or metastases, intracranial abscess, the patients with history of central nervous system infection, neurosurgery; the patients with benign intracranial hypertension, cranial trauma, pathology which causes jugular compression and those with findings of intoxication were excluded.

A trained emergency medicine specialist applied a thin layer of gel to both eyes of the patients in the supine position and performed the measurement with a $7.5 \mathrm{MHz}$ linear probe. The optic nerve sheath diameter was measured 
from $3 \mathrm{~mm}$ behind the posterior part of the globe from the sagittal and transverse planes at the same point. The left and right optic nerve sheath diameters were calculated by using the median value of the transverse and sagittal measurements. This calculation yielded the median optic nerve sheath diameter. All images were controlled and confirmed by a blinded emergency medicine specialist, subsequently, the patient and control groups were compared.

The widest ONSD appeared due to intracranial pressure increase was at $3 \mathrm{~mm}$ behind the globes; no significant dilatation was shown posterior than $3 \mathrm{~mm}$ [10]. Therefore, ONSD was measured at axial plane at $3 \mathrm{~mm}$ posterior to the optic nerve on both eyes from brain parenchyma frame; and average of the measurements was obtained.

The data were evaluated through SPSS (Statistical Package for Social Sciences) Windows 22.0 program. Distribution of the continuous variables was tested by Kolmogorov Smirnov test. The descriptive data were expressed in number of cases (n) and percentages $(\%)$. The quantitative data were presented in median and interquartile range (IQR). The continuous data were compared using the Mann Whitney-U, and the qualitative data were compared using the chi-square test. The relationship between the categorical variables and ONST were evaluated using the Spearman correlation analysis. An ROC curve was prepared to demonstrate the effectiveness of ONST in indicating ICP. The results were evaluated in a confidence interval of $95 \%$ and a significance level of $p<0.05$.

\section{Results}

The median age of the patients in our study was 71 (IQR: 19), and $51.3 \%$ of the subjects were males. The two groups were similar regarding age, gender, presence of additional diseases,
Table 1. Comparison of demographic characteristics of the patients and control group.

\begin{tabular}{|l|l|l|c|}
\hline Parameters & $\begin{array}{l}\text { CVA } \\
(\mathbf{n = 3 8 6})\end{array}$ & $\begin{array}{l}\text { Control } \\
(\mathbf{n}=\mathbf{7 5})\end{array}$ & $\boldsymbol{p}$ \\
\hline Age, Median (IQR) & $71(19)$ & $71(19)$ & 0,713 \\
\hline $\begin{array}{l}\text { Gender Male, n (\%) } \\
\text { Female, n (\%) }\end{array}$ & $\begin{array}{l}198(51.3) \\
188(48.7)\end{array}$ & $\begin{array}{l}39(52.0) \\
36(48.0)\end{array}$ & 0,911 \\
\hline OSNT, Median (IQR) & $5.25(0.25)$ & $4.1(0.25)$ & $<0,001$ \\
\hline $\begin{array}{l}\text { CCT OSNT, } \\
\text { Median (IQR) }\end{array}$ & $5.60(0.65)$ & & \\
\hline $\begin{array}{l}\text { Time (min), Median } \\
\text { (IQR) }\end{array}$ & $260(80)$ & & \\
\hline GCS, Median (IQR) & $15(0)$ & - & \\
\hline NIHSS, Median (IQR) & $12(7)$ & - & \\
\hline $\begin{array}{l}\text { Concomitant disease, } \\
\text { n (\%) }\end{array}$ & $292(75.6)$ & $49(65.3)$ & 0.063 \\
\hline HT, n (\%) & $235(60.9)$ & $46(61.3)$ & 0.941 \\
\hline DM, n (\%) & $118(30.6)$ & $13(17.3)$ & 0.020 \\
\hline CAD, n (\%) & $94(24.4)$ & $14(18.7)$ & 0.287 \\
\hline AF, n (\%) & $31(8.0)$ & $1(1.3 \%)$ & 0.037 \\
\hline Previous CVA, n (\%) & $27(7.0)$ & $3(4.0 \%)$ & 0.336 \\
\hline COPD/Asthma, n (\%) & $17(4.4)$ & $1(1.3 \%)$ & 0.209 \\
\hline CHF, n (\%) & $16(4.1)$ & 0 & 0.073 \\
\hline Other, n (\%) & $6(1.6)$ & 0 & 0.277 \\
\hline SBP, The Median (IQR) & $165(32)$ & $124(28)$ & $<0.001$ \\
\hline DBP, Median (IQR) & $90(20)$ & $80(20)$ & $<0.001$ \\
\hline $\begin{array}{l}\text { Pulse, The Median } \\
\text { (IQR) }\end{array}$ & $82(15)$ & $69(17)$ & $<0.001$ \\
\hline $\begin{array}{l}\text { Hospitalized in, Clinic } \\
\text { (\%) }\end{array}$ & $286(74.1)$ & & \\
\hline Death, n (\%) & $5.25(0.25)$ & $4.1(0.25)$ & $<0.001$ \\
\hline ONST & & & \\
\hline
\end{tabular}

$n$ : number of patients, ONST: nerve sheath thickness, CCT: Cranial computed tomography GCS: Glasgow Coma Scale, NIHSS: National Institute Of Health Sciences Scale, HT: Hypertension, DM: Diabetes Mellitus, CAD: Coronary Artery Disease, AF: Atrial Fibrillation, CVA: Cerebrovascular Accident, COPD: Chronic Obstructive Pulmonary Disease, CHF: Congestive Heart Failure, SBP: Systolic Blood Pressure, DBP: Diastolic Blood Pressure, ONST: Optic Nerve Sheath Thickness.

hypertension (HT), previous strokes, coronary artery disease (CAD), congestive heart failure (CHF), chronic obstructive pulmonary disease (COPD)/asthma, and other comorbidity rates 
Table 2. Stroke type and the findings.

\begin{tabular}{|c|c|c|}
\hline Type & Findings & $\begin{array}{l}\text { CVA } \\
(n=386)\end{array}$ \\
\hline \multirow[t]{4}{*}{$\begin{array}{l}\text { Haemorrhagic, } \\
\text { n (\%) } 34(8.8)\end{array}$} & $\begin{array}{l}\text { Intraparenchymal } \\
\text { hematoma }\end{array}$ & $18(4.7)$ \\
\hline & $\begin{array}{l}\text { Haemorrhage } \\
\text { opened into the } \\
\text { ventricle }\end{array}$ & $8(2.1)$ \\
\hline & $\begin{array}{l}\text { Non-traumatic } \\
\text { subarachnoid } \\
\text { haemorrhage }\end{array}$ & $8(2.1)$ \\
\hline & $\begin{array}{l}\text { Shift larger than } 3 \\
\mathrm{~mm}\end{array}$ & $11(2.8)$ \\
\hline \multirow[t]{6}{*}{$\begin{array}{l}\text { Ischemic, } \mathbf{n} \\
(\%)^{* * 352}(91.2)\end{array}$} & $\begin{array}{l}\text { Infarction of the } \\
\text { middle cerebral } \\
\text { artery }\end{array}$ & $190(49.2)$ \\
\hline & $\begin{array}{l}\text { Infarction of the } \\
\text { anterior cerebral } \\
\text { artery }\end{array}$ & $10(2.6)$ \\
\hline & $\begin{array}{l}\text { Vertebrobasillary } \\
\text { artery }\end{array}$ & $102(26.4)$ \\
\hline & Lacunar infarction & $86(22.3)$ \\
\hline & Undetected & $8(2.1)$ \\
\hline & $\begin{array}{l}\text { Shift larger than } \\
3 \mathrm{~mm}\end{array}$ & $22(5.7)$ \\
\hline
\end{tabular}

* Cerebral magnetic resonance imaging and tomography data were collected.

** Since one case has multiple lesions, number of total cases is higher than the patients with ischemic stroke.

( $p>0.05)$ (Table 1). In our study, the median ONST diameter value of the stroke patients was $5.25 \mathrm{~mm}$ median (IQR: 0.25), where the median ONST diameter of the control group was 4.1 mm (IQR: 0.25). The median ONST value of the stroke patients was significantly higher $(p<0.001)$. Median ONST diameter value of the stroke patients detected by CT was $5.60 \mathrm{~mm}$ median (IQR: 0.65). The period between clinical development and USS scan was 260 minutes (IQR: 80). The prevalence of diabetes mellitus (DM) and atrial fibrillation (AF) was significantly higher among the stroke patients $(p<0.05)$. The patient's median Glasgow Coma Scale (GCS) score was 15 (IQR: 0) and the median NIHSS score was 12 (IQR: 6.3). The systolic blood pressure (SBP), diastolic blood pressure (DBP) and pulse values were found to be higher for the study group compared to the control group $(p<0.05)$. $74.1 \%$ of the patients were hospitalized in the clinic and is $25.9 \%$ were hospitalized in the ICU, $6.2 \%$ of the patients died (Table 1).In the patient group, $91.2 \%$ of stroke patients were ischemic and $8.8 \%$ were hemorrhagic. The most common cause for ischemia was infarction of the middle cerebral artery; the most common cause for hemorrhage was parenchymal hematoma (Table 2).

The median ONST value was $5.5 \mathrm{~mm}$ (IQR: 0.30 ) for the hemorrhagic stroke patients, and $5.25 \mathrm{~mm}$ (IQR: 0.20) for the ischemic stroke patients. The ONST diameters of the hemorrhagic patients were found to be significantly higher $(p<0.001)$ (Figure 1).

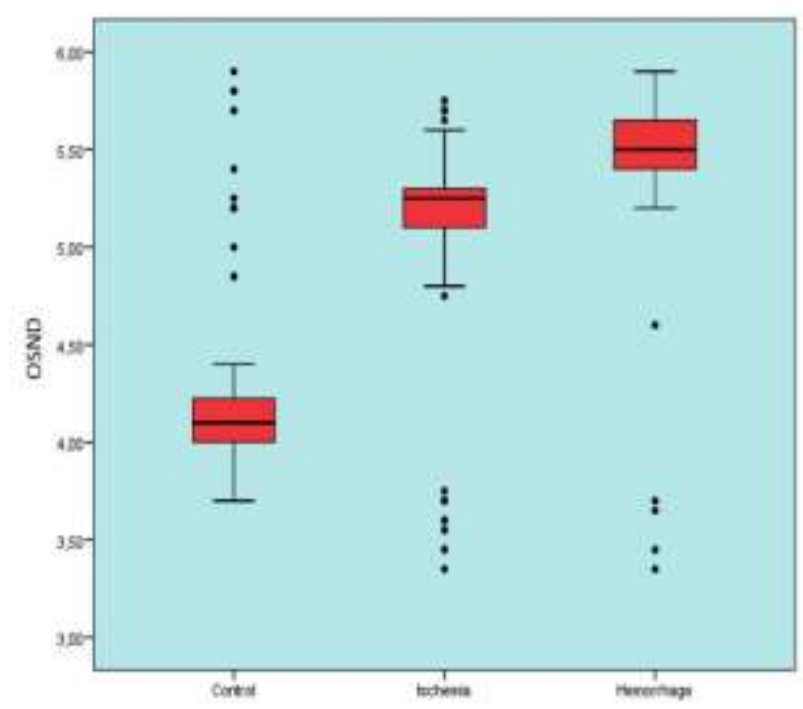

Figure 1. Comparison of the ONST groups.

In the present study (all groups including hemorrhagic and ischemic patients), there was no correlation between ONST diameter; age and pulse $(p>0.05)$. There was a negative correlation between ONST and GCS, and a positive correlation between ONST and ONST detected by CT, USS period, SBP, DBP, NIHSS $(p<0.05)$ (Table 3$)$. 
Table 3. The association between the ONST and demographic data.

\begin{tabular}{|l|l|l|l|l|l|l|}
\hline Data & \multicolumn{2}{|c|}{ All subjects } & \multicolumn{2}{l|}{ Haemorrhagic } & \multicolumn{2}{|l|}{ Ischemic } \\
\hline & $r$ & $p$ & $r$ & $p$ & $r$ & $p$ \\
\hline Age & 0.003 & 0.947 & 0.555 & 0.001 & -0.005 & 0.923 \\
\hline $\begin{array}{l}\text { CCT } \\
\text { ONST }\end{array}$ & 0.704 & $<0.001$ & 0.910 & $<0.001$ & 0.702 & $<0.001$ \\
\hline Time & 0.168 & 0.001 & 0.777 & $<0.001$ & 0.194 & $<0.001$ \\
\hline GCS & -0.294 & $<0.001$ & -0.668 & $<0.001$ & -0.171 & 0.001 \\
\hline NIHSS & 0.239 & $<0.001$ & 0.428 & 0.012 & 0.172 & 0.001 \\
\hline SBP & 0.207 & $<0.001$ & 0.685 & $<0.001$ & 0.121 & 0.023 \\
\hline DBP & 0.231 & $<0.001$ & 0.419 & 0.014 & 0.176 & 0.001 \\
\hline Pulse & 0.010 & 0.849 & -0.011 & 0.951 & 0.002 & 0.970 \\
\hline
\end{tabular}

CCT: Cranial computed tomography, GCS: Glasgow Coma Scale, NIHSS: National Institute of Health Sciences Scale, SBP: Systolic Blood Pressure, DBP: Diastolic Blood Pressure, ONST: Optic nerve sheath thickness

In this study, a significant correlation between ONST; and age, HT, DM, AF, CAD, previous CVA and COPD/asthma ( $p>0.05)$ was not detected. Also, it was determined that the ONST diameter increased with comorbidities and $\mathrm{CHF}(p<0.05)$.

The hospitalization unit (clinic/ICU) was not found to be correlated with ONST ( $p>0.05)$, however, the ONST values of the patients that died were significantly higher $(p<0,05)$ (Table 4).

A ROC curve was prepared to demonstrate the effectiveness of ONST in indicating ICP. The area under the curve has been detected to be $0.865 ; 4.5 \mathrm{~mm}$ cut-off value for $96.1 \%$, specificity was $82.7 \%$ sensitivity (Figure 2 ).
Table 4. The correlation between ONST and comorbidities, hospitalization unit and death.

\begin{tabular}{|l|c|c|c|}
\hline Parameters & Group 1 & Group 2 & $p$ \\
\hline Gender & $5.25(0.25)$ & $5.25(0.25)$ & 0.664 \\
\hline Comorbidity & $5.25(0.25)$ & $5.20(0.21)$ & 0.039 \\
\hline HT & $5.25(0.30)$ & $5.20(0.25)$ & 0.158 \\
\hline DM & $5.25(0.25)$ & $5.25(0.25)$ & 0.619 \\
\hline AF & $5.30(0.30)$ & $5.25(0.25)$ & 0.051 \\
\hline CAD & $5.25(0.21)$ & $5.25(0.25)$ & 0.809 \\
\hline Previous CVA & $5.30(0.35)$ & $5.25(0.25)$ & 0.088 \\
\hline COPD/Asthma & $5.25(0.42)$ & $5.25(0.25)$ & 0.404 \\
\hline CHF & $5.33(0.34)$ & $5.25(0.25)$ & 0.016 \\
\hline Other & $5.52(0.41)$ & $5.25(0.25)$ & 0.001 \\
\hline Outcome & $5.25(0.25)$ & $5.25(0.25)$ & 0.435 \\
\hline Death & $5.45(0.54)$ & $5.25(0.25)$ & 0.011 \\
\hline
\end{tabular}

(Group 1: male, present, clinic, died; Group 2: women, intensive care, alive) HT: Hypertension, DM: Diabetes Mellitus, CAD: Coronary Artery Disease, AF: Atrial Fibrillation, CVA: Cerebrovascular Accident, COPD: Chronic Obstructive Pulmonary Disease, CHF: Congestive Heart Failure.

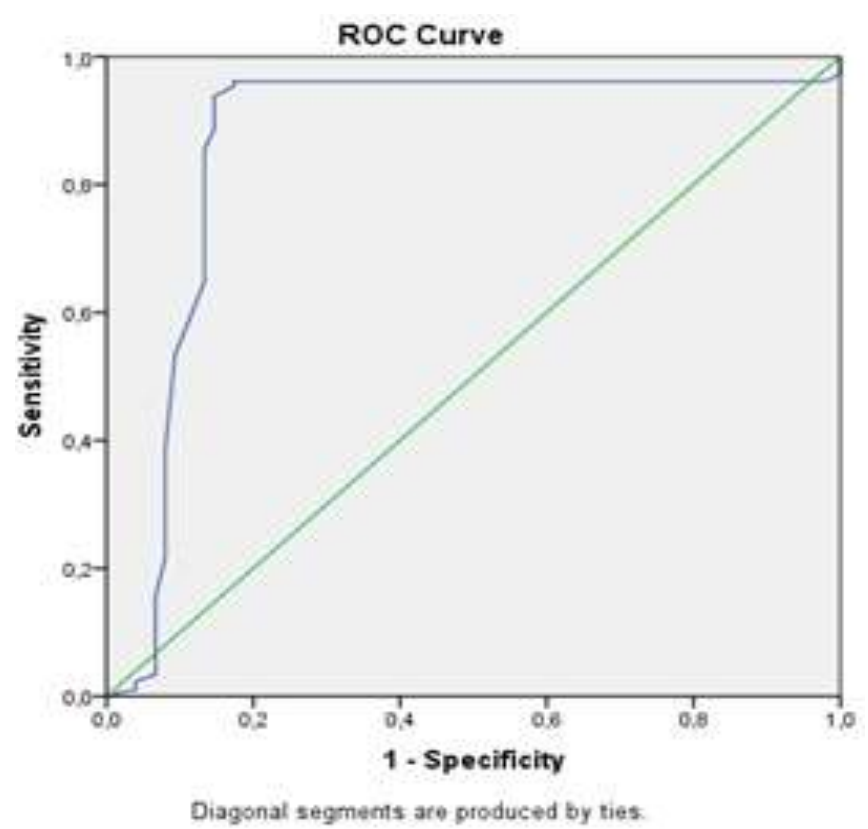

Figure 2. ONST diagnostic ROC curve. 


\section{Discussion}

The increase in ICP due to intracranial events can lead to the deterioration of cerebral perfusion, increase in ischemia and brain tamponed. Thus, it is accompanied by high mortality and mortality rates. Brain edema can be detected in the earlier stages and treated with brain edema decompression, which can decrease mortality from $78 \%$ to $29 \%$ [11]. The increased intracranial pressure should be reduced as quickly as possible $[12,13]$.

The relationship between ONST and ICP is contradictive. Kimberley et al. found a direct correlation between ONST and ICP [14]. Safak et al. reported that the diameter of ONST, measured through non-invasive methods, can be used to measure ICP [15]. However, it has been demonstrated that, in some special cases, the ONST doesn't increase despite increasing ICP. This is thought to be because the optic nerve sheath cannot expand in some patients, and due to the optic nerve sheath variations $[16,17]$.

Komut et al. found that mean ONST diameter was $5.4 \mathrm{~mm}$ in the patients with stroke, whereas this value was $4.1 \mathrm{~mm}$ for the control group. Such difference was found to be significant [4]. Yuzbasioglu et al. [18] found the mean ONST diameter to be $5.6 \mathrm{~mm}$ for stroke patients; this value was $3.6 \mathrm{~mm}$ for the control group. This difference was found to be significant. Gokcen et al. [6] indicate that the ONST diameter was higher for the stroke patients compared to the control group. Skoloudik et al. [16] indicated that there wasn't a significant difference between the ONST diameters of stroke patients and healthy controls; however, the median ONST value was significantly higher among hemorrhagic stroke patients. In our study, we found that the ONST diameter of the stroke patients was significantly higher. We believe that the increase in the ONST diameter is due to the primary events that occur due to hypoxia and the edema that develops due to the increased mediators.

Studies have found that $80-87 \%$ of all stroke cases are of is ischemic origin [2,3]. Chae et al. [19] indicate that the clinical condition of the hemorrhagic patients is worse compared to the ischemic stroke patients. Skoloudik et al. [16] have found that the ONST diameter was above $6.6 \mathrm{~mm}$ for $21 \%$ of the stroke patients, and above $5 \mathrm{~mm}$ for $71 \%$ of the patients. The same study found that the ONST diameter of $86 \%$ of hemorrhagic stroke patients was above $6.6 \mathrm{~mm}$. Komut et al. [4] have found that the ONST diameter was higher for the hemorrhagic patients compared to the ischemic patients; however, this difference was not statistically significant. In our study, the incidence of hemorrhagic stroke was $7.4 \%$, which is lower than the literature. It was also determined that the ONST diameter was higher for the hemorrhagic patients. This might be associated with the fact that; for patients who have hemorrhagic stroke, the bleeding can lead to more extensive damage, more significant disruption of the CSF circulation, and that there is more secondary damage.

Studies indicate that the patients with NIHSS and low GCS scores had higher mortality rates, and that these two scales can be used to predict clinical prognosis [20-22]. Komut et al. [4] found that the patients with lower GCS scores have higher ONST values. Yuzbasioglu et al. [20] found a positive correlation between NIHSS scores and ONST values. Jeng et al. [23] reported higher NIHSS scores and lower GCS scores among hemorrhagic stroke patients. In our study, we have found that ONST values were positively correlated with NIHSS scores and negatively correlated with GCS scores. It is known that increasing ICP worsens clinical condition. We believe that this 
outcome decreases the GCS score while increasing the NIHSS. We believe this is the reason why ONST is positively correlated with NIHSS, whereas it is negatively correlated with GCS.

High rates of hypertension and arrhythmias are reported for stroke patients. This is explained by the alteration of the sympathetic and parasympathetic efferent pathways from the cardio regulator centers of the brain stem due to the stroke [24-26]. Achieving cerebral autoregulation and decrease of cerebral parenchyma may cause an increase in the blood pressure [27]. Gokcen et al. [6] indicate that the stroke patients have elevated blood pressures. In our study, we found that the blood pressure and pulse values of the stroke patients had increased. A positive correlation was found between blood pressure and ONST; however, pulse rate was not found to be correlated with ONST. We believe that the impulses from the cardio-regulatory centers aim to increase the blood flow to the brain, thus increasing blood pressure and pulse. Since one of the components that determine ICP is blood pressure, we believe that a correlation occur between ONS and blood pressure. Furthermore, edema develops and blood pressure increases in proportion to hypoperfusion to prevent the hypoperfusion appeared due to irregular cerebral blood supply.

Studies indicate that comorbidities; such as DM, AF, HT, and previous SVO; may be risk factors for stroke, and that they increase the prevalence of strokes [23,28,29]. Complying with the literature, we have found the most common comorbidities in stroke patients to be HT (60.9\%) and DM (30.9\%); also, the prevalence of DM and AF had significantly increased for the patient group. In addition, ONST had significantly increased in patients with comorbidities and/or CHF. The most common pathologies may be HT and DM because these diseases are also the most common comorbidities. DM may increase the prevalence of stroke due to the disruption of vascular structures, and AF leads to increased rates of heart-related thrombi.

In his dissertation, Batur [30] found that the rate of increased intracranial pressure syndrome (IICPS) was higher among the patients that were hospitalized in the intensive care unit compared to the patients hospitalized in the clinics; however, this rate was lower than the patients who died. Legrand et al. [31] found that ONST was smaller among patients that survived traumatic brain damage. In our study, the ONST values of the patients that were hospitalized or that died were higher. This may be due to the fact that increased ICP worsens the clinical condition of the patients, and the physician decides to hospitalize the patient with a poor condition to the ICU. Also, the patients with increased ICP values have higher mortality rates.

The dysfunction of auto-regulation in cerebral blood flow in acute stroke cases causes secondary edema increase [27]. In the present study, a positive correlation was detected between USS period and ONST. We believe that such correlation appears due to further dysfunction of auto-regulation and eventual increase of edema and ICP increase because earlier interventions cannot be performed to the patient before clarification of the diagnosis.

Dadi et al. [32] conducted a study on the children with findings of increase in ICP and reported a positive correlation between ONST measured by USS and ONST measured by brain CT and MRI. The aforementioned study also stated that such association was stronger in the measurements performed within first 5 hours. Sekhon et al. [33] reported a strong positive correlation between cranial $\mathrm{CT}$ and 
ONST measured by USS in their study conducted on the cases with traumatic brain injury. In line with the literature, a strong correlation was detected between ONST detected by CT and USS in the present study. Batur [30] found in his dissertation that sensitivity of USG examinations was $95.7 \%$ for the IICPS-positive patients, whereas the specificity was $100 \%$. Gokcen et al. [6] found that, for the $5.3 \mathrm{~mm}$ cut-off value, the sensitivity was $80 \%$ and the specificity was $84 \%$. For the $4.7 \mathrm{~mm}$ cut-off value, the sensitivity was $70 \%$ and the specificity was $86 \%$. In our study, we have found that for the $4.5 \mathrm{~mm}$ cut-off values, sensitivity and specificity were determined to be $96.1 \%$ and $82.7 \%$, respectively. These values indicate that ONST can be a valuable parameter for the confirmation of the diagnosis of stroke patients and the indication of increased ICP.

The most important limitation of the present study is lack of control ONST measurements after follow-up and treatment. Further studies may be carried out on this topic. Another limitation is unclear time of the clinic presentation of the patient. This may be associated with unnoticeable clinical presentation at the beginning or attacks when the patients were alone or at sleep. However, many medical history data of the patients was not documented and added during history taking.

\section{Conclusion}

ONST values increase among stroke patients, and especially in patients with hemorrhagic strokes. If increase of ONST is detected in a patient, a treatment planning to reduce ICP may be considered.
Funding: There is no financial support and sponsorship

Conflict of Interest: The authors declare that they have no conflict of interest.

Ethical statement: The study was conducted in accordance with the ethical approval of the University Ethics Committee. (Date: 08/02/2018; Decision number: 2018/12).

\section{ORCID iD of the author(s)}

Kaan Celik / 0000-0002-9664-6732

Tamer Colak / 0000-0003-3844-4785

Beliz Oztok Tekten /0000-0002-4007-005X

\section{References}

[1]Yilmaz MS, Yilmaz M, Isik B, et al. The correlation of TWEAK level and with involved area on MRI in stroke. BioMed Res. 2017; 28(3):1110-14.

[2]Go S, Worman DJ. Stroke Syndromes in: Tintinalli JE (Eds), Emergency Medicine:A Comprehensive Study Guide (8th ed) Chapter 167. McGraw-Hill: 2015. p. 114256.

[3]Kumral E, Özkaya B, Sağduyu A, et al. The stroke registry. A hospital based study in the Aegian Region, İzmir, Tukey. Analysis of 2000 patients. Cerebrovasc Dis. 1998; 8(5):278-88.

[4]Komut E, Kozaci N, Sonmez BM, et al. Bedside sonographic measurement of optic nerve sheath diameter as a predictor of intracranial pressure in ED. Am J Emerg Med. 2016; 34(6):963-67.

[5]Shevlin C. Optic nerve sheath ultrasound for the bedside diagnosis of intracranial hypertension: pitfalls and potential. Cri Care Hor. 2015; 1(1):22-30.

[6]Gokcen E, Caltekin I, Savrun A, et al. Alterations in optic nerve sheath diameter according to cerebrovascular disease sub- 
groups. Am J Emerg Med. 2017; 35(11):1607-11.

[7]Hansen HC, Lagreze W, Krueger O, et al. Dependence of the optic nerve sheath diameter on acutely applied subarachnoidal pressure - an experimental ultrasound study. Acta Ophthalmol. 2011; 89(6):528-32.

[8]Amini A, Kariman H, Dolatabadi AA, et al. Use of the sonographic diameter of optic nerve sheath to estimate intracranial pressure. Am J Emerg Med. 2013; 31(1):236-39.

[9]Sahoo SS, Agrawal D. Correlation of optic nerve sheath diameter with intracranial pressure monitoring in patients with severe traumatic brain injury. The Ind J Neurot. 2013; 10(1):9-12.

[10]Hansen HC, Helmke K. The subarachnoid space surrounding the optic nerves. An ultrasound study of the optic nerve sheath. Surg Rad Anat. 1996; 18(4):323-28.

[11] Shapiro K, Marmarou A, Shulman K. Characterization of clinical CSF dynamics and neural axis compliance using the pressure-volume index: I. The normal pressure-volume index. Ann Neuro. 1980; 7(6):508-14.

[12] Shirodkar CG, Munta K, Rao SM, et al. Correlation of measurement of optic nerve sheath diameter using ultrasound with magnetic resonance imaging. Indian $\mathrm{J}$ Cri Care Med. 2015; 19(8):466-70.

[13] Soldatos T, Karakitsos D, Chatzimichail K, et al. Optic nerve sonography in the diagnostic evaluation of adult brain injury. Crit Care. 2008; 12(3):R67.

[14]Kimberly HH, Shah S, Marill K, et al. Correlation of optic nerve sheath diameter with direct measurement of intracranial pressure. Acad Emerg Med. 2008; 15(2):201-4.
[15] Şafak KY, Türkoğlu Ö, Şencan BD, et al. Optik Sinir Kılıfı Çapı Ölçümlerinde MRG ve USG Arasındaki Uyum. Okmeydanı Tip Der. 2015; 31(2):71-74.

[16] Školoudík D, Herzig R, Fadrná T, et al. Distal enlargement of the optic nerve sheath in the hyperacute stage of intracerebral haemorrhage. Brit J Ophthalmol. 2011; 95(2):217-21.

[17] Killer H, Jaggi G, Flammer $J$, et al. Cerebrospinal fluid dynamics between the intracranial and the subarachnoid space of the optic nerve. Is it always bidirectional? Brain. 2006; 130(2):514-20.

[18] Yuzbasioglu Y, Yuzbasioglu S, Coskun S, et al. Bedside measurement of the optic nerve sheath diameter with ultrasound in cerebrovascular disorders. Turk J Med Sci. 2018; 48(1):93-99.

[19] Chae J, Zorowitz RD, Johnston MV. Functional outcome of hemorrhagic and nonhemorrhagic stroke patients after inpatient rehabilitation: A Matched Comparison. Am J Phys Med \& Rehabil. 1996; 75(3):177-82.

[20] Johnston KC, Connors A, Wagner D, et al. A predictive risk model for outcomes of ischemic stroke. Stroke. 2000; 31(2):448-55.

[21]Collaboration GSS. Predicting outcome after acute ischemic stroke An external validation of prognostic models. Neurology. 2004; 62(4):581-85.

[22] Appelros P, Nydevik I, Viitanen M. Poor outcome after first-ever stroke: predictors for death, dependency, and recurrent stroke within the first year. Stroke. 2003; 34(1):122-26.

[23]Jeng J-S, Huang S-J, Tang S-C, et al. Predictors of survival and functional outcome in acute stroke patients admitted to the stroke intensive care unit. J Neurosci. 2008; 270(1-2):60-66. 
[24]Alioğlu Z, Bostan M, Alioğlu Y, et al. Primer Kalp Hastalığı Olmayan Akut Serebrovasküler Hastalıklarda 24 Saatlik Holter Monitörizasyonu. Turgut Özal Tip Mer Der. 1998; 5(1):40-42.

[25] Hacke W, Kaste M, Bluhmki E, et al. Thrombolysis with alteplase 3 to 4.5 hours after acute ischemic stroke. N Eng J Med. 2008; 359(13):1317-29.

[26] Lewandowski C, Barsan W. Treatment of acute ischemic stroke. Ann Emerg Med. 2001;37: 202-16.

[27] Minhas JS, Panerai RB, Ghaly G, et al. Cerebral autoregulation in hemorrhagic stroke: A systematic review and metaanalysis of transcranial Doppler ultrasonography studies. J Clin Ultrasound. 2019; 47(1):14-21.

[28] Andersen KK, Olsen TS, Dehlendorff C, et al. Hemorrhagic and ischemic strokes compared: stroke severity, mortality, and risk factors. Stroke. 2009; 40(6):2068-72.

[29] O'Donnell MJ, Xavier D, Liu L, et al. Risk factors for ischaemic and intracerebral haemorrhagic stroke in 22 countries (the INTERSTROKE study): a case-control study. The Lancet. 2010; 376(9735):112-23.

[30] Batur A. The diagnostic value of optic nerve sheath diameter measurements by ultrasonography in elevated intracranial pressure in stroke patients. http://www.openaccess.hacettepe.edu.tr:808 0/xmlui/handle/11655/3503.

[31]Legrand A, Jeanjean P, Delanghe F, et al. Estimation of optic nerve sheath diameter on an initial brain computed tomography scan can contribute prognostic information in traumatic brain injury patients. Crit Care. 2013; 17(2):R61.

[32]Dad1 B, Uyar E, Asadov R, et al. Comparison of Ultrasound Guided Optic Nerve Sheath Diameter Measurements with
Other Cranial Imaging Methods (Cranial Computed Tomography and Magnetic Resonance Imaging) in Pediatric Intensive Care Patients. J Pediatr Emerg Intensive Care Med. 2019; 6(1):1-6

[33] Sekhon MS, Griesdale DE, Robba C, et al. Optic nerve sheath diameter on computed tomography is correlated with simultaneously measured intracranial pressure in patients with severe traumatic brain injury. Intensive Care Med. 2014; 40(9):1267-74. 